\title{
All-Weather Microphones - Calibration and Uncertainty Evaluation Methods
}

\author{
G. WSZOŁEK* \\ Department of Mechanics and Vibroacoustics, AGH University of Science and Technology \\ al. A. Mickiewicza 30, 30-059 Kraków, Poland
}

\begin{abstract}
The paper presents the problems encountered during calibration of devices equipped with all-weather microphones, which results in frequent omissions in the periodic check-up of monitoring systems. Such a check-up should be regularly carried out, as it would result in broadening and unification of the knowledge concerning the measurements and the evaluation of their uncertainty in the environmental conditions.
\end{abstract}

PACS: 43.28.-g, 43.28. $+\mathrm{h}$

\section{Introduction}

According to the legally binding legal acts (regulations), in particular with Environment Protection Act there is a requirement of acoustic state assessment in the environment and elaboration of acoustic maps. Monitoring of sound level in the environment is carried out using monitoring stations, which are usually the measurement systems comprising of sound level meters equipped with the all-weather microphones. In absence of other legal regulations the systems for sound level measurement in the environment are being the subject to the procedures of legal and technical standard inspection similar to any other regular sound level meters with typical measurement microphones. They are subject to both legal metrological inspection - the requirement approval of type according to the respective government directive [1] as well as the requirements included in the PN-EN 61672 standard [2]. When the meters are used for measurements carried out by certified laboratories, they are also subject to periodic calibration.

The author of this paper has been working for many years in Accredited Laboratory which is calibrating devices for acoustic measurements. In practice measurement systems usually do not have type approval, are periodically calibrated only occasionally, and their current calibration by acoustic calibrators are often prepared improperly.

There is also an ample problem with uncertainty evaluation related to calibration of devices equipped with all-weather microphones. All-weather microphones are integral components of devices for their regular working mode in the environment (see Fig. 1). Uncertainty evaluation for environmental sound level measurements should contain sub-components, which are going to illustrate such factors like for e.g.:

- calibration and metrological characteristics of measurement system,

* e-mail: grazyna.wszolek@agh.edu.pl

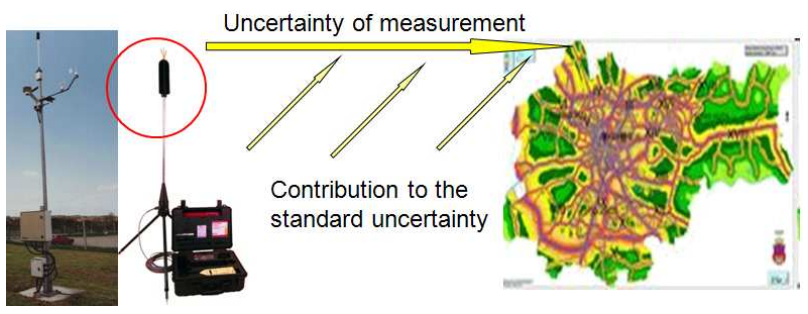

Fig. 1. Effect of various factors on the measurements of environmental noise.

- measurement methodology,

— weather factors influence,

- human factor (psychophysical features),

- results correlation with weather conditions and acoustic events.

All-weather microphones are the first, the most sensitive (in the sense of weak, unreliable, vulnerable) and the most important element in the measuring chain.

An important part is a quantitative evaluation of uncertainty components related to calibration, effects of correction and environmental conditions on all-weather microphones - even if the value of these factors is less important in comparison with other uncertainty components of environmental measurement results, like: wind speed and wind direction changes, background effects, sound level variation and possibly also human factors.

\section{Construction and properties of all-weather microphones}

Environmental noise monitoring systems in most cases consist of all-weather microphones with their preamplifiers, connected by cables to the measurement devices or computer data acquisition, analysis and/or transfer systems. In general the all-weather microphones can be divided according to several criteria.

The most important division criteria are:

— assembly (permanent or mobile), 
— operation time (continuous or periodic monitoring),

- the applied protective shielding of the microphone insert (typical all-weather microphones or all-weather protection housings mounted on typical measurement microphones),

- microphone direction, indicating the type of noise monitored (airport noise $-0^{\circ}$, environmental noise $\left.-90^{\circ}\right)$

- type of the microphone cartridge (externally-polarized or prepolarized microphone).

A complete all-weather microphone setup consists of a microphone, in which the microphone insert and the pre-amplifier form a single, integrated component (e.g. B\&K type 4952 microphone - see Fig. 2a), upon which the all-weather housing is mounted. A microphone setup that is more frequently used consists of a standard measurement microphone adapted to environmental measurements by attaching an all-weather housing, consisting in most cases of the following elements: microphone's protective grid in the all-weather version, rain-protection cap and a windscreen with wire ribs fixing the housing and driving away the birds. Another solution is the Svantek type SA 203 all-weather housing, in which the windscreen is also used as a waterproof rain cap.

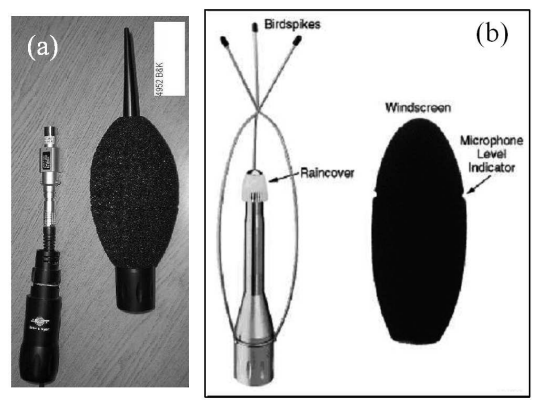

Fig. 2. Components of a typical all-weather microphone: (a) 4952 type all-weather microphone by Brüel \& Kjær, (b) UA-1404 type all-weather protection kit applied to a Brüel \& Kjær microphone [3].

Typical elements of an all-weather microphone (in addition to the microphone cartridge and preamplifier) irrespective whether this is a complete all-weather microphone or a measuring microphone adapted to all-weather conditions by a proper protection kit - are the windproof and waterproof shields and a special spiky construction against birds (see Fig. 2b). In most cases the adaptation of a measuring microphone is realized by replacing the standard microphone protection grid by a special protection grid dedicated for the all-weather versions and mounting the windproof and waterproof shields. In order to prevent water vapor condensation the microphone cartridges are always heated. The heating systems with additional water absorbers (dessicators), that contain a special drying medium (silica gel), prevent possible condensation of water vapor (see Fig. 3d).

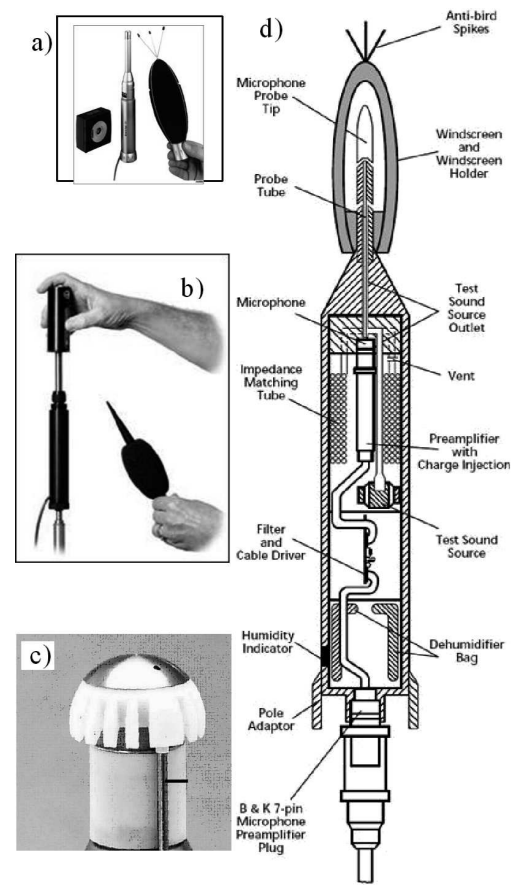

Fig. 3. Different types of measuring lines calibration with all-weather microphones $[3,5]$.

The output amplifiers allow the application of long output cables for connection of the all-weather measuring microphones with respective terminals (sound level meters or other dedicated measuring systems). For example the all-weather, permanent mode Norsonic Nor 1210 type microphone can be connected to a cable up to $500 \mathrm{~m}$ in length.

\section{The legal situation and metrological requirements applicable to all-weather microphones}

At the moment in Poland there are no uniform and clear legal regulations concerning the measuring devices for acoustic environmental measurements. There are no standards, government orders or directives or other recommendations regarding the requirements or testing procedures for all-weather microphones. The all-weather microphones, treated as additional equipment of measuring systems, are subject to the same testing procedures as other sound level meters, and the testing results should be referred to the requirements of the PN-EN 61672 standard [2]. Measuring systems with all-weather microphones should be periodically calibrated, at least once during every 24 months. They should also be subject to inter-laboratory measurement. Majority of meters available at the Polish market which are equipped with all-weather microphones do not have any calibration certificates.

Because of technical problems - which will be discussed below — in actual situation the measurement sys- 
tems with all-weather microphones are examined only at the pattern approval stage [4].

During calibrations carried out according to part 3 of the standard [2] the tests have to be carried out for a microphone attached to a measuring device. The characteristics are obtained by indirect methods - by calibration of the whole setup (without the shields) using the electrostatic actuator method (attached to the microphone's membrane) and application of necessary corrections for all sheltering elements forming the all-weather shield. Such calibrations are possible only for rare, selected types of monitoring systems. They are mainly measuring systems in which all-weather shields are used and for which the setup allows the calibration of the microphone together with the measuring device, using the electrostatic actuator method. Among setups that fulfill the condition there are: Svantek type SA 203, Sonopan type DSA-50 ZWP or B\&K type UA 1404. In theory these systems can be calibrated by the method described above, in practice for majority of these devices the manufacturing companies do not provide values of corrections, that should be applied to the characteristics determined using the electrostatic actuator. This is a necessary condition for making use of the indirect methods. One cannot calibrate by the indirect method devices like G.R.A.S. type 41AM or $41 \mathrm{CN}$ all-weather microphones [5]. This is prevented by the mechanical construction of the microphone, which contains its own electrostatic actuator used for remote calibration (see Fig. 3c). Microphones that cannot be calibrated by the electrostatic actuator have to be calibrated directly in the free field. Such a calibration requires a very specialized measurement stand, located in anechoic chamber, with proper extra fittings and a qualified personnel.

Therefore, in spite of various weather conditions in which the all-weather microphones are operated, various times of their installations at the monitoring stations, and also various microphone types, registering various sound characteristics (with respect to sound spectrum and incidence direction of the acoustic wave) the calibration operations of monitoring systems are not carried out. There are also no recommendations for formulation of unified uncertainty budgets for environmental noise measurement results. Most often even in the properly constructed uncertainty budgets there are no factors related to all-weather microphones, only the effects regarding the microphone insert are included [6]. This is a consequence of the absence of exhaustive information concerning the all-weather microphones. Some of the information should be included in the manufacturer's user manuals, according to respective regulations [1] and standards [2]. Other information should be provided - even if they are not required by law in the product documentation - because without that information it is not possible to determine the measurement error, when using the all-weather microphone.

This problem can be presented on the example of the Outdoor Microphone System Type 41 AM, by G.R.A.S.
Co., but similar problems are encountered for many products by other companies. The calibration card of the 41AM microphone contains data regarding calibration using electrostatic actuator $(90 \mathrm{~dB} \pm 0.2$, at frequency $1000 \mathrm{~Hz}$, with uncertainty $U=0.4 \mathrm{~dB}$ ) and the frequency characteristics. Because that microphone is the "airport noise" type microphone the characteristic presented in the calibration card is valid for wave incidence direction $0^{\circ}$ and it fulfills requirements of the standard [2]. The presented characteristic has been certainly obtained with proper filters, correcting the actual characteristics. The manufacturer does not publish - as it is typical for e.g. standard $1 / 2^{\prime \prime}$ microphones - the directivity characteristics and respective frequency dependent corrections for acoustic wave incidence directions other than $0^{\circ}$ (see Fig. 4).

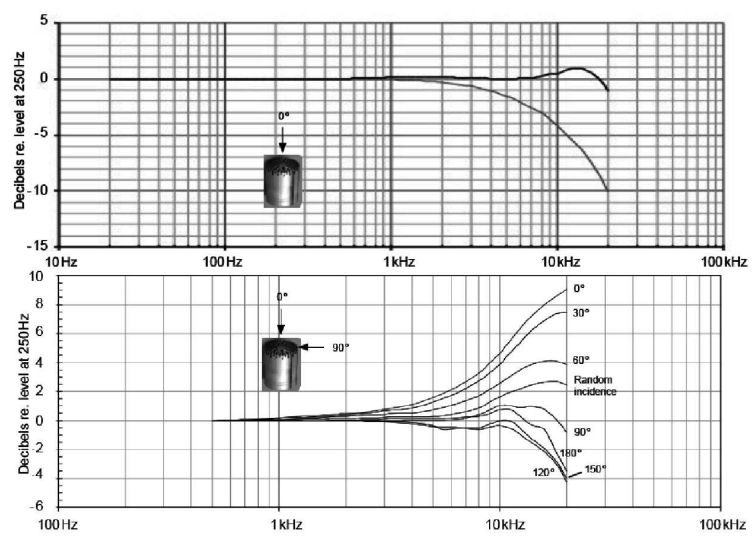

Fig. 4. Free field corrections for an exemplary $1 / 2^{\prime \prime} \mathrm{mi}-$ crophone - applied to the characteristics determined by the electrostatic actuator method for various incidence directions of the acoustic wave [7].

The specific nature of the aviation noise consists in the fact that such noise affects relatively wide terrain area, and the motion of the sound source results in sound wave propagation with predominantly zenithal incidence direction, but the exact momentary direction will depend on the exact location of the aircraft, and may be considerably different from the $0^{\circ}$ value (see Fig. 5).

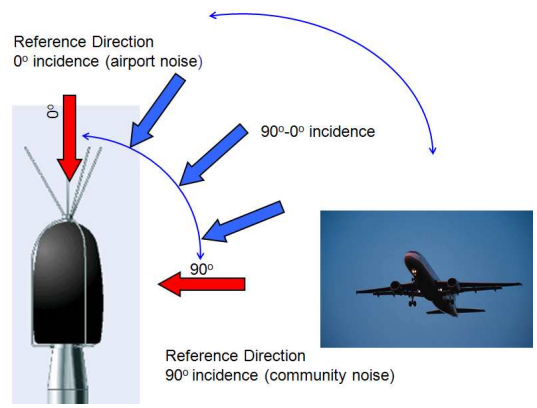

Fig. 5. The direction of incidence of the acoustic wave. 
The absence of full information regarding the behavior of the all-weather microphone both in frequency domain and the directivity characteristics does not allow the evaluation of errors resulting from variations of incidence direction for the acoustic wave reaching the microphone.

It is therefore important to learn how does the microphone behave as a function of frequency for other wave incidence directions, especially that the compensation filters are implemented for specific incidence directions $\left(0^{\circ}\right.$ and $/$ or $90^{\circ}$ ).

Figure 6 presents frequency characteristics measured at the Laboratory of Department of Mechanics and Vibroacoustics for a measuring device with all-weather microphone, without compensation filter and after its implementation. In the picture one can also find the values of acceptable errors, as required by the standard [2] (taking into account the maximum values of the extended measurement uncertainty).

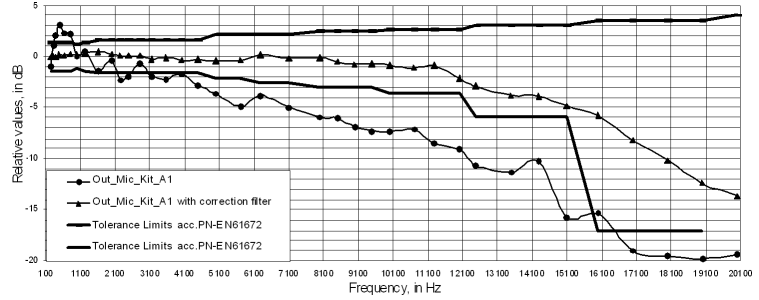

Fig. 6. Characteristics for a sound level meter with all-weather housing A1.

As can be seen from Fig. 6 the meter characteristics with the all-weather housing (for the $0^{\circ}$ incidence direction, as declared by the manufacturer), without the compensation filter are not conformant to the standard [2] requirements.

The manufacturers do not also inform how do the measurement results change as a consequence of all-weather shield performance e.g. falling rain or snow, snow or ice coverage. The only information that one can find is related to the recommended shield replacement periods (one or two years - depending on the manufacturer).

\section{Calibration of measuring lines}

Conducting environmental noise measurements by instruments using all-weather microphones includes a number of issues, including:

- field calibration of the measuring line (in the sense of: on-site of microphones installation),

- possibility of periodic calibration of measuring sets in accredited calibration laboratories,

- problems with the estimation of uncertainty component resulting from measurements performed with sound level meter equipped with all-weather microphone.
The existence of various construction schemes of all-weather microphones is the reason that for a proper calibration of a measuring line on-site a detailed knowledge of the user's manual is required and only the accessories dedicated to a given measuring system should be used, with the applied microphone, shielding and adapter types as specified by the manual. It is necessary because during the calibration one should take into account the free-field corrections provided by the manufacturer.

Calibration of the measuring lines is performed in the acoustic (direct) mode or the electrical (remote) mode (see Fig. 3). It covers mostly the calibration of measuring line at one point of frequency characteristics - for one frequency and for one sound level.

In cases when acoustic calibrators are applied the systems can be directly calibrated with all types of all-weather microphones. The acoustic calibrators are mounted directly on the microphone (after dismounting the all-weather protection shield as e.g. in B\&K and Svantek microphones) or additional adapters can be used, that allow exact matching of calibrator to the microphone (e.g. in microphones made by G.R.A.S.).

For microphones applied in continuous monitoring systems ("outdoor") the acoustic calibration is carried out from time to time. The systems are often calibrated remotely. The all-weather microphones for continuous monitoring contain an in-built acoustic actuator (often mounted inside the waterproof shield), which enables remote calibration of the measuring line by $1000 \mathrm{~Hz}$ signal from the internal generator, and in some microphone types the determination of the whole frequency characteristics of the measuring line by using an external generator. In the systems manufactured by $\mathrm{B} \& \mathrm{~K}$ a dedicated method is also used for measuring the output voltage at open microphone terminals with application of the charge injection calibration (CIC). In practice the method is often applied in multi-channel systems and in all situations when the microphone accessibility is difficult.

\section{Evaluating the uncertainty of measurement effect resulting from the all-weather microphone itself}

Even when the measurement is performed strictly in accordance with Guide to the Expression of Uncertainty in Measurement, in the final effect evaluation of measurement uncertainty always depends on actions of the person carrying out the measurements, his/her knowledge and experience. During evaluation of the measurement uncertainty one should take into account the metrological data obtained during examination of a given device type, and potential dependence of these parameters on time and ambient conditions. There are no guidelines which could specify which sources of error should be taken into account in the uncertainty budget.

The author believes that the main (dominant) sources of uncertainty of measurement effects resulting from all-weather microphone itself include: 
- calibrating by calibrator,

- correction because of all-weather housing (and all elements associated with it: a protection grid, rain cover, windshield, carrying structure),

- the influence of weather on the all-weather microphone,

- the influence of incidence direction for the acoustic wave reaching the microphone.

In the device type examination stage one determines the characteristics (together with the respective determination uncertainty), effects of additional accessories and the corrections applied during periodic tests using the electrostatic actuator and acoustic calibrator. The necessary data are usually found in the user's manual and the person evaluating the uncertainty of the measurements performed is able to make use of the data, provided that he/she possesses sufficient knowledge of the subject. The manuals usually do not provide the data concerning long-term changes of the parameters of all-weather microphones during prolonged environmental measurements. No data can be found either regarding the uncertainty of remote calibrations of measuring lines.

\section{Summary}

Environmental noise monitoring systems in most cases consist of all-weather microphones with their preamplifiers, connected by cables to the measurement devices or computer data acquisition/transfer and/or analysis systems.

Basing on experience and contacts with persons using monitoring systems the author noticed that there are a lot of problems connected with correct environmental measurements which are in practice concealed. In most cases the staff performing measurements do not have enough knowledge concerning proper calibration procedures of their measuring devices, i.e. using the necessary corrections, extracting the necessary data from user manual and other documentation. The manuals provided by the manufacturers very often do not contain all the necessary data. Even if they contain predominant part of the information, the manner in which it is presented makes the extraction rather difficult for a person lacking specialized knowledge.

The paper also presents the problems encountered during calibration of devices equipped with all-weather microphones, which results in frequent omissions in the periodic check-up of monitoring systems. Such a check-up should be regularly carried out, as it would result in broadening and unification of the knowledge concerning the measurements and the evaluation of their uncertainty in the environmental conditions.

Measuring systems with all-weather microphones should be periodically calibrated, at least once during every 24 months. They should also be subjected to inter-laboratory measurement. Periodic inspection and inter-laboratory testing contribute to deepen and consolidate knowledge about measurements and evaluation of their uncertainty in the environment.

Because measurement systems from various manufacturers are different, the level of knowledge of people performing measurements is also on different level. It is not possible to estimate the quantity of errors associated with incorrect instrument calibration as the same as judgement of measurement's uncertainty. To achieve this the analysis for particular instruments should be performed, including:

- calibration method at one point of characteristics (usually for frequency of $1000 \mathrm{~Hz}$ and for the level of 94 or $114 \mathrm{~dB}$ ),

- influence of using the appropriate compensation filter (airport noise or community noise),

- periodic calibration of instruments in accredited laboratories, including series of tests and entire frequency range in accordance with the third part of the standard [2],

- errors resulting from the influence of spectrum of measured sounds and direction they reach the microphones.

Due to the vastness of the problem it is not possible to perform such analysis in this article.

In the author's opinion these problems should be discussed and methods leading up to enlarging measurement accuracy and correctness of methods of received effects uncertainty evaluation should be jointly developed.

\section{References}

[1] Regulation by the Ministry of Economy dated 28th May, $2007 \mathrm{y}$. concerning the requirements imposed on the sound level meters and a detailed specification of examinations and check-ups carried out during legal metrological inspection of those measurement devices (Dz.U. nr 105, poz. 717).

[2] PN-EN 61672:2005, Electroacoustics - Sound Level Meters, Part 1, 2, 3.

[3] Brüel \& Kjær, Product Data Outdoor Microphone Kit $U A-1404$.

[4] G. Wszołek, in: The 14th Int. Conf. on Noise Control, Elblag (Poland), CIOP-PIB, Warszawa 2007.

[5] Instruction Manual Outdoor Microphone System Types $41 A M / 41 C N$.

[6] T. Wszołek in: Proc. XXXV Winter School of Vibroacoustic Hazards Control, Gliwice-Ustron, Ed. M. Roczniak, Polskie Towarzystwo Akustyczne, Oddział Górnośląski, Gliwice 2006, p. 205 (in Polish).

[7] Internet pages of: Brüel \& Kjær, G.R.A.S., Norsonic, Sonopan, and Svantek. 